\title{
Index and secondary index theory for flat bundles with duality
}

\author{
Ulrich Bunke*and Xiaonan $\mathrm{Ma}^{\dagger}$
}

February 8, 2020

\begin{abstract}
We discuss some aspects of index and secondary index theory for flat bundles with duality. This theory was first developed by Lott [9]. Our main purpose in the present paper is provide a modification with better functorial properties.
\end{abstract}

\section{Contents}

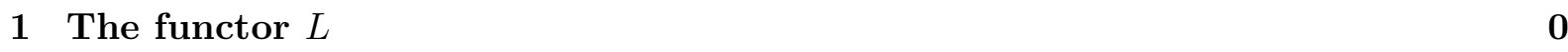

$1.1 \quad$ Definition of $L \ldots \ldots \ldots \ldots \ldots \ldots \ldots \ldots$

1.2 The natural transformation to $K$-theory $\ldots \ldots \ldots \ldots \ldots \ldots \ldots$. . . . . . . . 2

1.3 Secondary $L$-groups. The $\mathbb{R} / \mathbb{Z}$-variant $\ldots \ldots \ldots \ldots \ldots \ldots$. . . . . . . . . . . .

1.4 Index and secondary index $\ldots \ldots \ldots \ldots \ldots \ldots \ldots$

2 Geometry and extended secondary L-groups 5

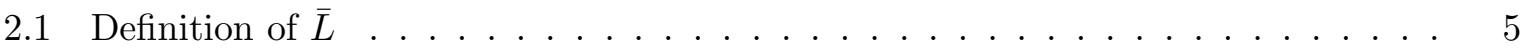

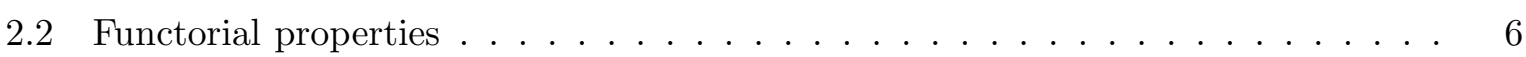

\begin{tabular}{|lll}
\hline 3 & Eta invariants and index maps & 7
\end{tabular}

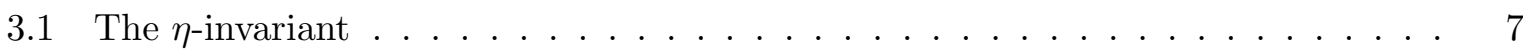

3.2 The secondary index $\operatorname{map} \ldots \ldots \ldots \ldots \ldots \ldots$

3.3 The index map for $L^{e x}$ and $L^{e x} \ldots \ldots \ldots \ldots \ldots \ldots \ldots$

\section{The functor $L$}

*Göttingen, bunke@uni-math.gwdg.de

${ }^{\dagger}$ Berlin, ma@mathematik.hu-berlin.de 


\section{$1.1 \quad$ Definition of $L$}

We define a contravariant functor $L$ from the category $\mathcal{T}$ op of topological spaces and continuous maps to $\mathbb{Z}_{2}$-graded rings. Given $X \in O b(\mathcal{T} o p)$ we define $L(X)$ by generators and relations.

If $\mathcal{F}$ is a locally constant sheaf of finite-dimensional $\mathbb{R}$-modules over $X$, then an $\epsilon$-symmetric duality structure on $\mathcal{F}$ is an isomorphism of sheafs $q: \mathcal{F} \rightarrow \mathcal{F}^{*}$ satisfying $q^{*}=(-1)^{\epsilon} q$, where $\mathcal{F}^{*}:=\operatorname{Hom}(\mathcal{F}, \underline{\mathbb{R}})$.

A generator of the group $L_{\epsilon}(X)$ is a pair $(\mathcal{F}, q)$ consisting of a locally constant sheaf of finitedimensional $\mathbb{R}$-modules and an $\epsilon$-symmetric duality structure $q$.

The relations in $L_{\epsilon}(X)$ are generated by the following:

1. isomorphism $(\mathcal{F}, q) \sim\left(\mathcal{F}^{\prime}, q^{\prime}\right)$ if there is an isomorphism of $\mathcal{F}$ with $\mathcal{F}^{\prime}$ which is compatible with the duality structures.

2. $\operatorname{direct} \operatorname{sum}(\mathcal{F}, q)+\left(\mathcal{F}^{\prime}, q^{\prime}\right) \sim\left(\mathcal{F} \oplus \mathcal{F}^{\prime}, q \oplus q^{\prime}\right)$

3. isotropic reduction Let $(\mathcal{F}, q)$ be a generator of $L(X)$ and $i: \mathcal{L} \hookrightarrow \mathcal{F}$ be a locally constant isotropic subsheaf, i.e. the composition $\mathcal{L} \stackrel{i}{\rightarrow} \mathcal{F} \stackrel{q}{\rightarrow} \mathcal{F}^{*} \stackrel{i^{*}}{\rightarrow} \mathcal{L}^{*}$ vanishes. Then $\mathcal{L}^{\perp}:=\operatorname{ker}\left(i^{*} \circ q\right)$ is a locally constant sheaf of finite-dimensional $\mathbb{R}$-modules containing $\mathcal{L}$. The quotient $\mathcal{F}_{\mathcal{L}}:=\mathcal{L}^{\perp} / \mathcal{L}$ comes equipped with an induced duality structure $q_{\mathcal{L}}$, and the pair $\left(\mathcal{F}_{\mathcal{L}}, q_{\mathcal{L}}\right)$ is called the isotropic reduction of $(\mathcal{F}, q)$ with respect to $\mathcal{L}$. In $L_{\epsilon}(X)$ we require

$$
(\mathcal{F}, q) \sim\left(\mathcal{F}_{\mathcal{L}}, q_{\mathcal{L}}\right)
$$

The class of $(\mathcal{F}, q)$ in $L_{\epsilon}(X)$ will be denoted by $[\mathcal{F}, q]$. The ring structure $L_{\epsilon}(X) \otimes L_{\epsilon^{\prime}}(X) \rightarrow$ $L_{\epsilon+\epsilon^{\prime}}(X)$ is induced by the tensor product:

$$
[\mathcal{F}, q]\left[\mathcal{F}^{\prime}, q \prime\right]:=\frac{\sqrt{(-1)^{\epsilon}} \sqrt{(-1)^{\epsilon^{\prime}}}}{\sqrt{(-1)^{\epsilon+\epsilon^{\prime}}}}\left[\mathcal{F} \otimes \mathcal{F}^{\prime}, q \otimes q^{\prime}\right] .
$$

The sign-convention is made such that later we have a natural transformation of rings from $L$ to complex $K$-theory $K^{0}$.

If $f: Y \rightarrow X$ is a morphism in $\mathcal{T} o p$, then $f^{*}: L(X) \rightarrow L(Y)$ is defined by $f^{*}[\mathcal{F}, q]=\left[f^{*} \mathcal{F}, f^{*} q\right]$. The map $f^{*}$ only depends on the homotopy class of $f$.

Remark: A version $L(X)^{\text {Lott }}$ of this ring was first introduced by Lott [9]. His definition differs from our since ours relation "isotropic reduction" is repaced by "hyperbolic is trivial" in the definition of Lott. Here a generator $(\mathcal{F}, q)$ is called hyperbolic if there is an isotropic subsheaf $\mathcal{L} \subset \mathcal{F}$ such that $\mathcal{F}_{\mathcal{L}}=0$ and such that $\mathcal{L}$ admits a complement inside $\mathcal{F}$. In particular, $L(X)$ is a quotient of $L(X)^{\text {Lott }}$. 


\subsection{The natural transformation to $K$-theory}

By $\mathcal{T} o p_{\text {para }}$ we denote the full subcategory of $\mathcal{T}$ op of paracompact topological spaces. Let $K^{0}(X)$ the complex $K$-theory functor. We construct a natural transformation $b: L \rightarrow K^{0}$ of functors from $\mathcal{T}$ op para to rings.

A locally constant sheaf of finite-dimensional $\mathbb{R}$-modules on $X$ gives rise to a locally trivial real vector bundle bundle $(\mathcal{F})$ in a natural way. The correspondence bundle is functorial and compatible with direct sum, tensor product, and duality. Thus applying the bundle construction to $(\mathcal{F}, q)$ we obtain a pair $(F, Q)$ consisting of a finite-dimensional real vector bundle and an isomorphism $Q: F \rightarrow F^{*}$.

Let $(F, Q)$ be a real vector bundle with an isomorphism $Q: F \rightarrow F^{*}$ such that $Q^{*}=(-1)^{\epsilon} Q$ for $\epsilon \in \mathbb{Z}_{2}$. Following the language introduced by Lott [9] we call an isomorphism $J: F \rightarrow F$ a metric structure, if

1. $J^{*} \circ Q$ defines a scalar product on $F$,

2. $J^{2}=\epsilon \operatorname{id}_{F}$,

3. $J^{*} \circ Q=(-1)^{\epsilon} Q \circ J$.

Since we assume that $X$ is paracompact it admits partitions of unity. This implies that metric structures exist, and that the space of all metric structures is contractible.

Given $(F, Q)$ as above we choose a metric structure $J$. Let $F_{\mathbb{C}}$ be the complexification of $F$. Then $\frac{1}{\sqrt{(-1)^{\epsilon}}} J$ is a $\mathbb{Z}_{2}$-grading of $F_{\mathbb{C}}$, and the pair $\left(F_{\mathbb{C}}, \frac{1}{\sqrt{(-1)^{\epsilon}}} J\right)$ represents an element of $K_{0}(X)$ which does not depend on the choice of $J$.

The transformation $b: L \rightarrow K^{0}$ is obtained by composing the latter construction with bundle.

\subsection{Secondary $L$-groups. The $\mathbb{R} / \mathbb{Z}$-variant}

We first recall the definition of the 2-periodic cohomology theory $K_{\mathbb{R} / \mathbb{Z}}$ introduced by [7], [8]. Let $B U$ be the classifying space of complex $K$-theory. The Chern character (with real coefficients) is

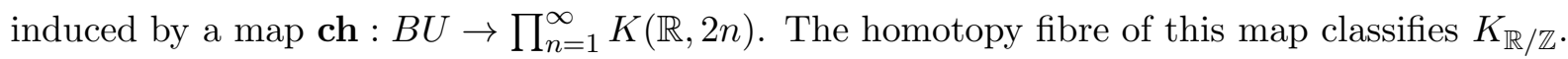
In particular, for any paracompact space $X$ there is a natural exact sequence of $K^{0}(X)$-modules

$$
\rightarrow K^{-1}(X) \stackrel{\text { ch }}{\rightarrow} H^{\text {odd }}(X, \mathbb{R}) \rightarrow K_{\mathbb{R} / \mathbb{Z}}^{-1}(X) \stackrel{\beta}{\rightarrow} K^{0}(X) \stackrel{\text { ch }}{\rightarrow} H^{e v}(X, \mathbb{R}) \rightarrow
$$

where $K^{0}(X)$ acts on cohomology via the Chern character.

We now define the functor $X \mapsto \bar{L}^{\mathbb{R} / \mathbb{Z}}(X)$ from paracompact topological spaces to $\mathbb{Z}_{2}$-graded 
groups by the following pull-back diagram:

$$
\begin{array}{ccc}
\bar{L}^{\mathbb{R} / \mathbb{Z}}(X) & \rightarrow & L(X) \\
\downarrow & & \downarrow b \\
K_{\mathbb{R} / \mathbb{Z}}^{-1}(X) & \stackrel{\beta}{\rightarrow} & K^{0}(X)
\end{array} .
$$

The grading is induced from that of $L(X)$. On morphisms the functor $L^{\mathbb{R} / \mathbb{Z}}$ only depends on homotopy classes. Note that $K^{0}(X)$ and $K_{\mathbb{R} / \mathbb{Z}}^{-1}(X)$ are $L(X)$-modules via $b$. This induces a graded $L(X)$-module structure on $\bar{L}^{\mathbb{R} / \mathbb{Z}}(X)$. We have the following natural exact sequence of $L(X)$-modules

$$
K^{-1}(X) \stackrel{\text { ch }}{\rightarrow} H^{\text {odd }}(X, \mathbb{R}) \rightarrow \bar{L}^{\mathbb{R} / \mathbb{Z}}(X) \rightarrow L(X) \stackrel{\text { chob }}{\rightarrow} H^{e v}(X, \mathbb{R})
$$

\subsection{Index and secondary index}

Let $X \rightarrow B$ be a smooth locally trivial fibre bundle over a compact base $B$ with compact evendimensional fibres such that the vertical bundle $T X / B$ is orientable. In this case we have the following maps:

- $\pi_{*}^{\text {sign }}: H^{*}(X, \mathbb{R}) \rightarrow H^{*}(B)$ defined by $\pi_{*}(\omega)=\int_{X / B} \omega \cap \mathbf{L}(T X / B)$, where $\int_{X / B}$ is integration over the fibre and $\mathbf{L}(T X / B)$ denotes the Hirzebruch $\mathbf{L}$-class of the vertical bundle.

- $\pi_{!}^{\text {sign }}: K^{0}(X, \mathbb{R}) \rightarrow K^{0}(B)$ defined by $\pi^{\text {sign }}([E])=\operatorname{index}\left(D_{E}^{\text {sign }}\right)$, where $D_{E}^{\text {sign }}$ is the fibrewise signature operator twisted by $E$ and $\operatorname{index}\left(D_{E}^{\text {sign }}\right) \in K^{0}(B)$ denotes the class of the index bundle.

- $\pi_{*}^{L}: L(X) \rightarrow L(B)$ defined by $\pi_{*}^{L}[\mathcal{F}, q]=\left[H^{\bullet} R \pi_{*} \mathcal{F}, \pi_{*}(q)\right]$, where $H^{\bullet} R \pi_{*} \mathcal{F}=\oplus_{i=0}^{\infty} R^{i} \pi_{*}(\mathcal{F})$ is the direct sum of higher derived direct images of $\mathcal{F}$. The orientation of $T X / B$ provides an isomorphism $p: R^{\operatorname{dim}(T X / Z)} \pi_{*} \underline{\mathbb{R}} \stackrel{\sim}{\rightarrow} \underline{\mathbb{R}}$. It induces isomorphisms $R^{i} \pi_{*} \mathcal{F}^{*} \rightarrow$ $\left(R^{\operatorname{dim}(T X / Z)-i} \pi_{*} \mathcal{F}\right)^{*}$, and $\pi_{*}(q)$ is the composition of the sum of these maps with the sum of $R^{i} \pi_{*}(q): R^{i} \pi_{*} \mathcal{F} \rightarrow R^{i} \pi_{*} \mathcal{F}^{*}$.

It is an excercise in spectral sequences to show that $\pi_{*}^{L}$ is well-defined.

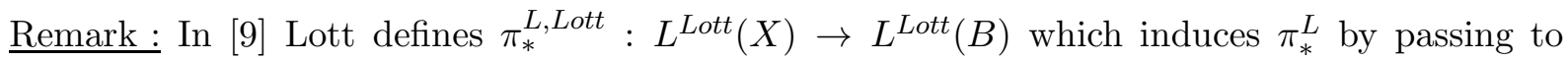
quotients.

By the index theorem for families and fibrewise Hodge theory the following diagram commutes

$$
\begin{aligned}
& L(X) \rightarrow K^{0}(X) \rightarrow H^{e v}(X, \mathbb{R}) \\
& \pi_{*}^{L} \downarrow \quad \pi_{!}^{\text {sign }} \downarrow \quad \pi_{*}^{\text {sign }} \downarrow . \\
& L(B) \rightarrow K^{0}(B) \rightarrow H^{e v}(B, \mathbb{R})
\end{aligned}
$$


In order to define an index map for $K_{\mathbb{R} / \mathbb{Z}}$ we need the further assumption that $\pi$ is $K$-oriented. Thus assume that $T X / B$ has a $\operatorname{Spin}_{c}$-structure. Then there are maps $\pi_{!}^{\operatorname{Spin}_{c}}: K^{0}(X) \rightarrow K^{0}(B)$ and $\pi_{!}^{\text {Spin }_{c}, \mathbb{R} / \mathbb{Z}}: K_{\mathbb{R} / \mathbb{Z}}^{-1}(X) \rightarrow K_{\mathbb{R} / \mathbb{Z}}^{-1}(B)$ (compare e.g. [8]), such that the following diagram commutes:

$$
\begin{array}{cccccccc}
H^{\text {odd }}(X, \mathbb{R}) & \rightarrow & K_{\mathbb{R} / \mathbb{Z}}^{-1}(X) & \rightarrow & K^{0}(X) & \rightarrow & H^{\text {ev }}(X, \mathbb{R}) \\
\pi_{*}^{\text {Spin }_{c}} \downarrow & & \pi_{!}^{\operatorname{Spin}_{c}, \mathbb{R} / \mathbb{Z}} \downarrow & & \pi_{!}^{\operatorname{Spin}_{c}} \downarrow & & \pi_{*}^{\text {Spin }_{c}} \downarrow \\
H^{\text {odd }}(B, \mathbb{R}) & \rightarrow & K_{\mathbb{R} / \mathbb{Z}}^{-1}(B) & \rightarrow & K^{0}(B) & \rightarrow & H^{\text {ev }}(B, \mathbb{R})
\end{array},
$$

where $\pi_{*}^{\operatorname{Spin}_{c}}(\omega)=\int_{X / Z} \hat{\mathbf{A}}(T X / B) \cap \mathrm{e}^{c_{1} / 2} \cap \omega$ and $c_{1}$ is the first Chern class determined by the

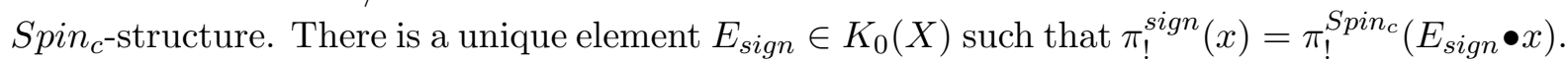
Note that $\operatorname{ch}\left(E_{\text {sign }}\right) \cap \hat{\mathbf{A}}(T X / B) \cap \mathrm{e}^{c_{1} / 2}=\mathbf{L}(T X / B)$.

- $\pi^{\text {sign }, \mathbb{R} / \mathbb{Z}}: K_{\mathbb{R} / \mathbb{Z}}^{-1}(X) \rightarrow K_{\mathbb{R} / \mathbb{Z}}^{-1}(B)$ is defined by $\pi^{\text {sign }, \mathbb{R} / \mathbb{Z}}(x)=\pi^{S p i n_{c}, \mathbb{R} / \mathbb{Z}}\left(E_{\text {sign }} \bullet x\right)$ so that the following diagram commutes

$$
\begin{array}{cccccccc}
H^{\text {odd }}(X, \mathbb{R}) & \rightarrow & K_{\mathbb{R} / \mathbb{Z}}^{-1}(X) & \rightarrow & K^{0}(X) & \rightarrow & H^{e v}(X, \mathbb{R}) \\
\pi_{*}^{\text {sign }} \downarrow & & \pi_{!}^{\text {sign, } / \mathbb{Z}} \downarrow & & \pi_{!}^{\text {sign }} \downarrow & & \pi_{*}^{\text {sign }} \downarrow \\
H^{\text {odd }}(B, \mathbb{R}) & \rightarrow & K_{\mathbb{R} / \mathbb{Z}}^{-1}(B) & \rightarrow & K^{0}(B) & \rightarrow & H^{e v}(B, \mathbb{R})
\end{array} .
$$

- $\pi_{*}^{\bar{L}, \mathbb{R} / \mathbb{Z}}: \bar{L}^{\mathbb{R} / \mathbb{Z}}(X) \rightarrow \bar{L}^{\mathbb{R} / \mathbb{Z}}(B)$ is the map induced by $\pi_{!}^{\text {sign, } \mathbb{R} / \mathbb{Z}}$ and $\pi_{*}^{L}$. The following diagramm commutes:

$$
\begin{array}{ccccccc}
H^{\text {odd }}(X, \mathbb{R}) & \rightarrow & \bar{L}^{\mathbb{R} / \mathbb{Z}}(X) & \rightarrow & L(X) & \rightarrow & H^{\text {ev }}(X, \mathbb{R}) \\
\pi_{*}^{\text {sign }} \downarrow & & \pi_{*}^{\bar{L}, \mathbb{R} / \mathbb{Z}} \downarrow & & \pi_{*}^{L} \downarrow & & \pi_{*}^{\text {sign }} \downarrow \\
H^{\text {odd }}(B, \mathbb{R}) & \rightarrow & \bar{L}^{\mathbb{R} / \mathbb{Z}}(B) & \rightarrow & L(B) & \rightarrow & H^{\text {ev }}(B, \mathbb{R})
\end{array} .
$$

All index maps are natural with respect to pull-back of fibre bundles.

Let $\pi_{1}: X \rightarrow X_{1}$ and $\pi_{2}: X_{1} \rightarrow B$ be locally trivial smooth fibre bundles with closed evendimensional fibres and compact base. Further assume that the vertical bundles $T X_{1} / X_{2}$ and $T X_{2} / B$ carry $\operatorname{Spin}_{c^{-}}$structures (and are therefore oriented). Then the composition $\pi=\pi_{2} \circ \pi_{1}$ : $X \rightarrow B$ is a locally trivial fibre bundle with closed even-dimensional fibres, and the vertical bundle $T X / B$ carries an induced $S_{p i n}$-structure. In this situation the index maps on complex

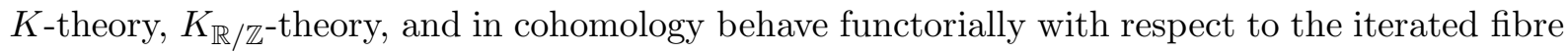
bundle. It is again an excercise in spectral sequences to show that $\pi_{*}^{L}$ is also functorial. As a consequence, $\pi_{*}^{\bar{L}, \mathbb{R} / \mathbb{Z}}$ is functorial, i.e. $\pi_{*}^{\bar{L}, \mathbb{R} / \mathbb{Z}}=\left(\pi_{2}^{\bar{L}, \mathbb{R} / \mathbb{Z}}\right)_{*} \circ\left(\pi_{1}^{\bar{L}, \mathbb{R} / \mathbb{Z}}\right)_{*}$.

Remark: The motivation of introducing the quotient $L(X)$ of Lott's group $L^{\text {Lott }}(X)$ is to have this functoriality for $\pi_{*}^{L}$, which is not true for Lott's definition. 


\section{Geometry and extended secondary $L$-groups}

\section{$2.1 \quad$ Definition of $\bar{L}$}

The functor $X \mapsto \bar{L}^{\mathbb{R} / \mathbb{Z}}(X)$ from $\mathcal{T} o p_{\text {para }}$ to $\mathbb{Z}_{2}$-graded $L(X)$-modules was defined by a purely homotopy-theoretic construction as an extension of the functor $X \mapsto \operatorname{ker}\left(\operatorname{ch} \circ b: L(X) \rightarrow H^{e v}(X, \mathbb{R})\right)$ by $X \mapsto H^{\text {odd }}(X, \mathbb{R}) / \mathbf{c h}\left(K^{-1}(X)\right)$.

Let $\mathcal{T} o p_{\text {smooth }}$ denote the full subcategory of topological spaces $\mathcal{T}$ op para which are homotopy equivalent to smooth manifolds. In the present section we use a differential geometric construction in order to define on $\mathcal{T} o p_{\text {smooth }}$ a functor $X \mapsto \bar{L}(X)$ to graded $L(X)$-modules which extends $X \mapsto \operatorname{ker}(\mathbf{c h} \circ b)$ by $X \mapsto H^{\text {odd }}(X, \mathbb{R}) / V(X)$, where $V(X) \subset \operatorname{ch}\left(K^{-1}(X)\right) \subset H^{\text {odd }}(X, \mathbb{R})$ is a certain $L(X)$-submodule depending functorially on $X$. In general it seems to be an interesting problem to compute $V(X)$.

First we define $\bar{L}(M)$ for a smooth manifold $M$. We define $\bar{L}(M)$ by generators and relations. A generator of $L_{\epsilon}(M)$ is a tuple $(\mathcal{F}, q, J, \rho)$, where $(\mathcal{F}, q)$ is a generator of $L_{\epsilon}(M), J$ is a metric structure on $F:=$ bundle $(\mathcal{F})$, and $\rho \in \Omega^{4 *-(-1)^{\epsilon}}(M) / \operatorname{im}(d)$ satisfies $d \omega=p\left(\nabla^{F}, J\right)$. Here $\left(\Omega^{*}(M), d\right)$ is the de real Rham complex of $M$ and $\nabla^{F}$ is the canonical flat connection on $F$ (such that $\operatorname{ker}\left(\nabla^{F}\right) \cong \mathcal{F}$ ). The metric structure $J$ induces $\mathbb{Z}_{2}$-grading of $F_{\mathbb{C}}$, and if $\nabla^{F_{\mathbb{C}}, J}$ denotes the even part of the extension of $\nabla^{F}$ to $F_{\mathbb{C}}$, then $p\left(\nabla^{F}, J\right):=\operatorname{ch}\left(\nabla^{F_{\mathbb{C}}, J}\right)=\operatorname{tr}_{s} \exp \left(-R^{\nabla^{F_{\mathbb{C}}, e v}} / 2 \pi \mathrm{i}\right)$ is the characteristic form representing $\mathbf{c h} \circ b([\mathcal{F}, q])$.

The relations in $L_{\epsilon}(M)$ are generated by:

1. isomorphism $(\mathcal{F}, q, J, \rho) \sim\left(\mathcal{F}^{\prime}, q^{\prime}, J^{\prime}, \rho^{\prime}\right)$ if there is an isomorphism $\mathcal{F} \rightarrow \mathcal{F}^{\prime}$ which is compatible with duality and metric structures, and $\rho=\rho^{\prime}$.

2. variation of metric structure $(\mathcal{F}, q, J, \rho) \sim\left(\mathcal{F}, q, J^{\prime}, \rho^{\prime}\right)$, if $\rho^{\prime}=\rho+\tilde{p}\left(\nabla^{F}, J^{\prime}, J\right)$, where $\tilde{p}\left(\nabla^{F}, J^{\prime}, J\right):=\tilde{\mathbf{c h}}\left(\nabla^{F_{\mathbb{C}}, J^{\prime}}, \nabla^{F_{\mathbb{C}}, J}\right)$ is the transgession Chern form such that $d \tilde{p}\left(\nabla^{F}, J^{\prime}, J\right)=$ $p\left(\nabla^{F}, J^{\prime}\right)-p\left(\nabla^{F}, J\right)$ (see e.g. [9]) for more details.

3. direct $\operatorname{sum}(\mathcal{F}, q, J, \rho)+\left(\mathcal{F}^{\prime}, q^{\prime}, J^{\prime}, \rho^{\prime}\right) \sim\left(\mathcal{F} \oplus \mathcal{F}^{\prime}, q \oplus q^{\prime}, J \oplus J^{\prime}, \rho+\rho^{\prime}\right)$

4. isotropic reduction Let $\mathcal{L} \subset \mathcal{F}$ be an isotropic locally constant subsheaf and $L:=$ bundle $(\mathcal{L})$. It induces a filtration $L \subset L^{\perp} \subset F$ by flat subbundles. The composition $J^{*} \circ Q$ induces a scalar product $h^{F}$ on $F$. Let $H$ be the orthogonal complement of $L$ inside $L^{\perp}$ with respect to $h^{F}$. Then we get an orthogonal decomposition $F=L \oplus H \oplus J(L)$. Let $\nabla^{\oplus}$ be the part of $\nabla^{F_{\mathbb{C}}}$ which preserves this decomposition. Then $J$ is parallel with respect to $\nabla^{\oplus}$, and $d \mathbf{c h}\left(\nabla^{\oplus}, \nabla^{F_{\mathbb{C}}}\right)=\mathbf{c h}\left(\nabla^{\left(F_{\mathcal{L}}\right)_{\mathbb{C}}}\right)-\mathbf{c h}\left(\nabla^{F_{\mathbb{C}}}\right)$. In $\bar{L}_{\epsilon}(M)$ we require $(\mathcal{F}, q, J, \rho) \sim\left(\mathcal{F}_{\mathcal{L}}, q_{\mathcal{L}}, J_{\mathcal{L}}, \rho^{\prime}\right)$, where $\rho^{\prime}=\rho+\tilde{\mathbf{c h}}\left(\nabla^{\oplus}, \nabla^{F_{\mathbb{C}}}\right)$ and $J_{\mathcal{L}}$ is the restriction of $J$ to $H \cong \operatorname{bundle}\left(\mathcal{F}_{\mathcal{L}}\right)$. 
By $[\mathcal{F}, q, J, \rho]$ we denote the class in $\bar{L}$ represented by $(\mathcal{F}, q, J, \rho)$.

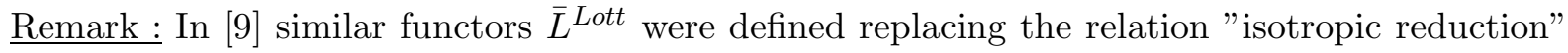
by "hyperbolic generators are trivial". Since Lott's relation is smaller than ours we have a natural surjective map $\bar{L}^{\text {Lott }}(M) \rightarrow \bar{L}(M)$.

The ring structure of $\bar{L}(X)$ over $L(X)$ is defined by $[\mathcal{F}, q, J, \rho] \bullet[\mathcal{E}, p]:=\left[\mathcal{F} \otimes \mathcal{E}, q \otimes p, J \otimes J^{E}, \rho \wedge\right.$ $\left.\operatorname{ch}\left(\nabla^{E_{\mathbb{C}}}\right)\right]$, where $J^{E}$ is any metric structure on bundle $(\mathcal{E})$.

\section{$2.2 \quad$ Functorial properties}

If $f: M \rightarrow N$ is a smooth map of manifolds, then we obtain an induced map $f^{*}: \bar{L}(N) \rightarrow \bar{L}(M)$, which is given on generators by pull-back of structures. $f^{*}$ only depends on the smooth homotopy class of $f$ and is compatible with the $L(M)$ resp. $L(N)$-module structures.

We define natural maps $H^{\text {odd }}(M, \mathbb{R}) \rightarrow \bar{L}(M)$ and $\bar{L}(M) \rightarrow L(M)$ by $[\rho] \mapsto[0,0,0, \rho]$ and $[\mathcal{F}, q, J, \rho] \mapsto[\mathcal{F}, q]$. Then we have the following exact sequence of $L(M)$-modules

$$
H^{\text {odd }}(M, \mathbb{R}) \rightarrow \bar{L}(M) \rightarrow L(M) \rightarrow H^{e v}(M, \mathbb{R})
$$

(see [9], Prop. 21, for a similar argument).

We now construct a natural morphism of $L(M)$-modules $\bar{\gamma}: \bar{L}(M) \rightarrow K_{\mathbb{R} / Z}^{-1}(M)$. Here we use the definition of $K_{\mathbb{R} / Z}^{-1}(M)$ in terms of generators and relations given in $[8]$, Def. $5+6$. A generator of $K_{\mathbb{R} / Z}^{-1}(M)$ is a tuple $\left(E, h^{E}, \nabla^{E}, \rho\right)$, where $E$ is a $\mathbb{Z}_{2}$-graded complex vector bundle of vertual dimension zero, $h^{E}$ is a hermitean metric and $\nabla^{E}$ is a metric connection, both being compatible with the grading, and $\rho \in \Omega^{\text {odd }}(M) / \operatorname{im}(d)$ satisfies $d \rho=\operatorname{ch}\left(\nabla^{E}\right)$. The relations of $K_{\mathbb{R} / Z}^{-1}(M)$ are generated by

1. isomorphism $\left(E, h^{E}, \nabla^{E}, \rho\right) \sim\left(E^{\prime}, h^{E^{\prime}}, \nabla^{E^{\prime}}, \rho\right)$ if there exists an isomorphism from $E$ to $E^{\prime}$ which is compatible with metrics and connections.

2. direct $\operatorname{sum}\left(E, h^{E}, \nabla^{E}, \rho\right)+\left(E^{\prime}, h^{E^{\prime}}, \nabla^{E^{\prime}}, \rho^{\prime}\right)=\left(E \oplus E^{\prime}, h^{E \oplus E^{\prime}}, \nabla^{E \oplus E^{\prime}}, \rho+\rho^{\prime}\right)$

3. change of connections $\left(E, h^{E}, \nabla, \rho\right) \sim\left(E, h^{E}, \nabla^{\prime}, \rho^{\prime}\right)$ if $\rho^{\prime}=\rho+\mathbf{c h}\left(\nabla^{\prime}, \nabla\right)$.

4. trivial elements If $\left(E, h^{E}, \nabla^{E}\right)$ is a $Z_{2}$-graded hermitean vector bundle with connection, then $\left(E \oplus E^{o p}, h^{E \oplus E}, \nabla^{E \oplus E}, 0\right) \sim 0$, where $E^{o p}$ denotes $E$ with the opposite grading.

Let $\left[E, h^{E}, \nabla^{E}, \rho\right]$ denote the class of $\left(E, h^{E}, \nabla^{E}, \rho\right)$ in $K_{\mathbb{R} / Z}^{-1}(M)$.

We define $\bar{L}(M) \rightarrow K_{\mathbb{R} / Z}^{-1}(M)$ by $\bar{\gamma}[\mathcal{F}, q, J, \rho]=\left[F_{\mathbb{C}}, h^{F_{\mathbb{C}}}, \nabla^{F_{\mathbb{C}}}, \rho\right]$, where $h^{F_{\mathbb{C}}}$ is the hermitean extension of the metric $J^{*} \circ Q$ on $F$. 
The following diagram commutes

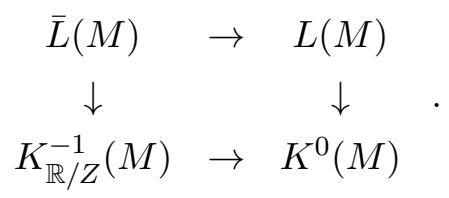

We therefore obtain a natural map $\bar{L}(M) \rightarrow \bar{L}^{\mathbb{R} / \mathbb{Z}}(M)$ which is in fact surjective.

We now extend the functor $\bar{L}$ to $\mathcal{T} o p_{\text {smooth }}$ by setting

$$
\bar{L}(X):=\lim _{f: \overleftarrow{M \rightarrow X}} \bar{L}(M)
$$

where the limit is taken over the category of manifolds over $X$. This extension has all functorial properties discussed above.

\section{Eta invariants and index maps}

\subsection{The $\eta$-invariant}

Using the $\eta$-invariant of the twisted signature operator for a closed odd-dimensional oriented manifold $M$ in [9] Lott constructed a group homomorphism $\eta^{\text {Lott }}: \bar{L}^{\text {Lott }}(M) \rightarrow \mathbb{R}$. Its reduction modulo $\mathbb{Z}$ factors over the homomorphism $\eta^{\mathbb{R} / \mathbb{Z}}: K_{\mathbb{R} / \mathbb{Z}}^{-1}(M) \rightarrow \mathbb{R} / \mathbb{Z}$ constructed in $\mathbb{8}$, which is given by the pairing with the $K$-homology class induced by the odd signature operator.

Unfortunately, the homomorphism $\eta^{\text {Lott }}$ does not factor over the quotient $\bar{L}^{\text {Lott }}(M) \rightarrow \bar{L}(M)$. In order to fix this for a given closed odd-dimensional oriented manifold $M$ we define an extension

$$
0 \rightarrow \mathbb{Z} \rightarrow \bar{L}_{\epsilon}^{e x}(M) \rightarrow L_{\epsilon}(M) \rightarrow 0
$$

such that

$$
\bar{\eta}: \bar{L}_{\epsilon}^{e x}(M) \rightarrow \bar{L}_{\epsilon}(M) \rightarrow K_{\mathbb{R} / \mathbb{Z}}^{-1}(M) \stackrel{\eta^{\mathbb{R} / \mathbb{Z}}}{\rightarrow} \mathbb{R} / \mathbb{Z}
$$

lifts to $\eta: \bar{L}_{\epsilon}^{e x}(M) \rightarrow \mathbb{Z}$. Note that $\bar{L}^{e x}(M)$ is not a functor on $M$. Here $n:=\operatorname{dim}(M)$, $\epsilon_{n}:=\left[\frac{n(n-1)}{2}\right] \in \mathbb{Z}_{2}$, and we assume that $\epsilon=1-\epsilon_{n}$.

Let $(\mathcal{F}, q)$ be a generator of $L_{\epsilon}(M)$ and $\mathcal{L}$ be an isotropic locally constant subsheaf of $\mathcal{F}$. We define an integer $\tau(\mathcal{F}, q, \mathcal{L})$ by the following construction. We consider the complex of sheaves

$$
\mathcal{K}^{\bullet}: \mathcal{L} \stackrel{i}{\rightarrow} \mathcal{F} \stackrel{i^{*} \circ q}{\rightarrow} \mathcal{L}^{*}
$$

Let $\left(E_{r}^{\bullet \bullet}, d_{r}\right), r \geq 1$ be the associated hyper cohomology spectral sequence. The duality $q$ and the pairing between $\mathcal{L}$ and $\mathcal{L}^{*}$ induce a duality $q_{\mathcal{K}}: \mathcal{K} \stackrel{\sim}{\rightarrow} \mathcal{K}^{*}[-2]$. The orientation of $M$ and $q$ induce a duality $q_{E_{r}}: E_{r} \stackrel{\sim}{\rightarrow} E_{r}^{*}[-2,-\operatorname{dim}(M)]$. Let $N_{r}$ denote the total grading on $E_{r}$. Then 
we define the symmetric form $Q_{r}$ on $E_{r}$ by $Q_{r}(v, w)=q_{E_{r}}\left((-1)^{\frac{N_{r}\left(N_{r}-1\right)}{2}} v\right)\left(d_{r} w\right)$. The integer $\tau(\mathcal{F}, q, \mathcal{L})$ is now given by

$$
\tau(\mathcal{F}, q, \mathcal{L}):=2\left(\operatorname{sign}\left(Q_{1}\right)+\operatorname{sign}\left(Q_{2}\right)\right)
$$

We first define an extension $L_{\epsilon}^{e x}(M)$ of $L_{\epsilon}(M)$ by generators and relations. A generator of the group $L_{\epsilon}^{e x}(M)$ is a triple $(\mathcal{F}, q, z)$ consisting of a locally constant sheaf of finite-dimensional $\mathbb{R}$-modules, an $\epsilon$-symmetric duality structure $q$, and an integer $z$.

The relations in $L_{\epsilon}^{e x}(M)$ are generated by the following.

1. isomorphism $(\mathcal{F}, q, z) \sim\left(\mathcal{F}^{\prime}, q^{\prime}, z\right)$ if there is an isomorphism of $\mathcal{F}$ with $\mathcal{F}^{\prime}$ which is compatible with the duality structures.

2. $\operatorname{direct} \operatorname{sum}(\mathcal{F}, q, z)+\left(\mathcal{F}^{\prime}, q^{\prime}, z^{\prime}\right) \sim\left(\mathcal{F} \oplus \mathcal{F}^{\prime}, q \oplus q^{\prime}, z+z^{\prime}\right)$

\section{3. isotropic reduction}

$$
(\mathcal{F}, q, z) \sim\left(\mathcal{F}_{\mathcal{L}}, q_{\mathcal{L}}, z+\tau(\mathcal{F}, q, \mathcal{L})\right)
$$

There is an exact sequence

$$
0 \rightarrow \mathbb{Z} \rightarrow L_{\epsilon}^{e x}(M) \rightarrow L_{\epsilon}(M) \rightarrow 0
$$

where the maps are the obvious inclusion and projection. We now define $\bar{L}_{\epsilon}^{e x}(M)$ by the following pull-back diagram:

$$
\begin{array}{ccc}
\bar{L}_{\epsilon}^{e x}(M) & \rightarrow & L_{\epsilon}^{e x}(M) \\
\downarrow & & \downarrow \\
\bar{L}_{\epsilon}(M) & \rightarrow & L_{\epsilon}(M)
\end{array} .
$$

Then we have exact sequences

$$
0 \rightarrow \mathbb{Z} \rightarrow \bar{L}_{\epsilon}^{e x}(M) \rightarrow \bar{L}_{\epsilon}(M) \rightarrow 0
$$

and

$$
H^{4 *-(-1)^{\epsilon}}(M, \mathbb{R}) \rightarrow \bar{L}_{\epsilon}^{e x}(M) \rightarrow L_{\epsilon}^{e x}(M) \rightarrow H^{4 *+1-(-1)^{\epsilon}}(M, \mathbb{R})
$$

and a natural way.

An element of $\bar{L}_{\epsilon}^{e x}(M)$ can be represented by a tuple $\left(\mathcal{F}, q, J^{F}, \rho, z\right)$. We obtain a class $\left[\mathcal{F}, q, J^{F}, \rho\right] \in$ $\bar{L}_{\epsilon}^{\text {Lott }}(M)$. If we set

$$
\eta\left(\mathcal{F}, q, J^{F}, \rho, z\right):=\eta^{\operatorname{Lott}}\left(\left[\mathcal{F}, q, J^{F}, \rho\right]\right)-z
$$

then we have the following result. 
Theorem 3.1 The map $\left(\mathcal{F}, q, J^{F}, \rho, z, g^{F}\right) \mapsto \eta\left(\mathcal{F}, q, J^{F}, \rho, z\right)$ induces a well-defined homomorphism $\eta: \bar{L}_{\epsilon}^{e x}(M) \rightarrow \mathbb{R}$ which lifts $\bar{\eta}$.

We must show that $\eta$ is well-defined with respect to isotropic reduction. The idea is to consider the $\eta$-invariant of the signature operator on $M$ twisted with the complex $K=$ bundle $(\mathcal{K})$ and to rescale its differential. The corresponding adiabatic limits can be understood by the methods developed in [3] and [6] without investing essentially new ideas.

\subsection{The secondary index map}

Let $M \rightarrow B$ be a smooth locally trivial fibre bundle with even-dimensional closed fibres over a compact base $B$ such that the vertical bundle $T M / B$ is oriented. We set $n:=\operatorname{dim}(T M / B)$.

In [9] Lott constructed a secondary index map $\pi_{*}^{\bar{L}, \text { Lott }}: \bar{L}_{\epsilon}^{\text {Lott }}(M) \rightarrow L_{\epsilon+\epsilon_{n}}^{\text {Lott }}(B)$ which fits into the commutative diagram

$$
\begin{aligned}
& H^{4 *-(-1)^{\epsilon}}(M, \mathbb{R}) \quad \rightarrow \quad \bar{L}_{\epsilon}^{\text {Lott }}(M) \rightarrow \bar{L}_{\epsilon}^{\mathbb{R} / \mathbb{Z}}(M) \\
& \pi_{*}^{\text {sign }} \downarrow \quad \pi_{*}^{\bar{L}, \text { Lott }} \downarrow \quad \pi_{*}^{\bar{L}, \mathbb{R} / \mathbb{Z} \downarrow .} \\
& H^{4 *-(-1)^{\epsilon+\epsilon_{n}}}(M, \mathbb{R}) \rightarrow \bar{L}_{\epsilon+\epsilon_{n}}^{\text {Lott }}(B) \rightarrow \bar{L}_{\epsilon+\epsilon_{n}}^{\mathbb{R} / \mathbb{Z}}(B)
\end{aligned}
$$

Theorem 3.2 By passing to quotients the map $\pi_{*}^{\bar{L}, \text { Lott }}$ induces a well-defined secondary index map

$$
\pi_{*}^{\bar{L}}: \bar{L}_{\epsilon}(M) \rightarrow L_{\epsilon+\epsilon_{n}}(B)
$$

The idea of the proof consists in investigating the adiabatic limits of the $\eta$-form of the fibrewise signature operator twisted with the complex $K$ under rescaling the differential. The arguments are similar to the case of analytic torsion forms. It suffices to adapt the methods developed in [1], 迆, [2], 10], 11], [12], 13].

The following diagram commutes

$$
\begin{aligned}
& H^{4 *-(-1)^{\epsilon}}(M, \mathbb{R}) \quad \rightarrow \quad \bar{L}_{\epsilon}(M) \quad \rightarrow \quad L_{\epsilon}(M) \\
& \pi_{*}^{\text {sign }} \downarrow \quad \pi_{*}^{\bar{L}} \downarrow \quad \pi_{*}^{L} \downarrow . \\
& H^{4 *-(-1)^{\epsilon+\epsilon_{n}}}(M, \mathbb{R}) \rightarrow \bar{L}_{\epsilon+\epsilon_{n}}(B) \rightarrow L_{\epsilon+\epsilon_{n}}(B)
\end{aligned}
$$

The secondary index map is natural with respect to pull-back of fibre bundles. Furthermore, if $\pi_{1}: X \rightarrow X_{1}$ and $\pi_{2}: X_{2} \rightarrow B$ is an iterated bundle with oriented vertical bundles, and $\pi=\pi_{2} \circ \pi_{1}$, then

Theorem $3.3 \pi_{*}^{\bar{L}}=\left(\pi_{2}^{\bar{L}}\right)_{*} \circ\left(\pi_{1}^{\bar{L}}\right)_{*}$

This follows from the functoriality of the $\eta$-form studied in [11] in a similar way as the functoriality of the secondary index in [5] was deduced from the functoriality of the higher analytic torsion form [10]. 


\subsection{The index map for $L^{e x}$ and $\bar{L}^{e x}$}

Let $\pi: M \rightarrow B$ as above and assume that $B$ is oriented, closed, of dimension $m$. Furthermore, assume that $\epsilon=1-\epsilon_{n}-\epsilon_{m}$. Let $(\mathcal{F}, q)$ be a generator of $L_{\epsilon}(M)$. We define an integer $\tau(\mathcal{F}, q, M \stackrel{\pi}{\rightarrow} B)$ by the following construction. Let $\left(E^{\bullet \bullet}, d_{r}\right)$ be the Leray-Serre spectral sequence converging to sheaf cohomology $H^{*}(M, \mathcal{F})$ with $E_{2}^{p, q}=H^{p}\left(B, R^{q} \pi_{*} \mathcal{F}\right)$. The orientations of $T M / B$ and $B$ and the duality $q$ induce dualities $q_{E_{r}}: E_{r} \rightarrow E_{r}^{*}[-m,-n]$ which give the Poincaré duality on the limit. We define the symmetric form $Q_{r}$ on $E_{r}$ by $Q_{r}(v, w)=q_{E_{r}}\left((-1)^{\frac{N_{r}\left(N_{r}-1\right)}{2}} v\right)\left(d_{r} w\right)$, where $N_{r}$ denotes the total degree. Then we set

$$
\tau(\mathcal{F}, q, M \stackrel{\pi}{\rightarrow} B):=2 \sum_{r \geq 2} \operatorname{sign}\left(Q_{r}\right) .
$$

Theorem $3.4 \quad$ 1. The prescription

$$
\pi_{*}^{L^{e x}}[\mathcal{F}, q, z]:=\left[H^{\bullet} R \pi_{*} \mathcal{F}, \pi_{*}(q), z-\tau(\mathcal{F}, q, M \stackrel{\pi}{\rightarrow} B)\right] .
$$

defines an extended index map $\pi_{*}^{L^{e x}}: L_{\epsilon}^{e x}(M) \rightarrow L_{\epsilon+\epsilon_{n}}^{e x}(B)$.

2. The extented index map is functorial with respect to iterated fibre bundles.

The following diagram

$$
\begin{array}{cccccc}
\bar{L}_{\epsilon}(M) & \rightarrow & L_{\epsilon}(M) & \leftarrow & L_{\epsilon}^{e x}(M) \\
\pi_{*}^{\bar{L}} \downarrow & & \pi_{*}^{L} \downarrow & & \pi_{*}^{L^{e x}} \downarrow \\
\bar{L}_{\epsilon+\epsilon_{n}}(B) & \rightarrow & L_{\epsilon+\epsilon_{n}}(B) & \leftarrow & L_{\epsilon+\epsilon_{n}}^{e x}(B)
\end{array}
$$

commutes and induces an extended secondary index map $\pi_{*}^{\bar{L}^{e x}}: \bar{L}^{e x}(M)_{\epsilon} \rightarrow \bar{L}_{\epsilon+\epsilon_{n}}^{e x}(B)$ which is functorial with respect to iterated fibre bundles. We have the following compatibility of this extended secondary index map with the $\eta$-homomorphism.

\section{Theorem 3.5}

$$
\begin{array}{cccc}
L_{\epsilon}^{e x}(M) & \stackrel{\eta}{\rightarrow} & \mathbb{R} \\
\pi_{*}^{\bar{L}^{e x}} \downarrow & & & \| \\
\bar{L}_{\epsilon+\epsilon_{n}}^{e x}(B) & \stackrel{\eta}{\rightarrow} & \mathbb{R}
\end{array}
$$

This theorem essentially follows from Dai's adabatic limit formula for the $\eta$-invariant [6].

\section{References}

[1] A. Berthomieu and J.M. Bismut. Quillen metrics and higher analytic torsion forms. J. Reine Angew. Math., 457(1994), 85-184. 
[2] J. M. Bismut. Families of immersions, and higher analytic torsion. Asterisque, 244, 1997.

[3] J. M. Bismut and J. Cheeger. $\eta$-invariants and their adiabatic limits. J. AMS, 2(1989), $33-70$.

[4] J.M. Bismut and G. Lebeau. Complex immersions and Quillen metrics. Publ. Math. IHES, 74, 1991.

[5] U. Bunke. On the functoriality of Lott's secondary analytic index. Preprint, 2000.

[6] X. Dai. Adiabatic limits, non-multiplicativity of signature and Leray spectral sequence. J. A.M.S., 4(1991), 265-321.

[7] M. Karoubi. Homologie cyclique et K-théorie. Asterisque, 149, 1987.

[8] J. Lott. R/Z-index theory. Comm. Anal. Geom., 2(1994), 279-311.

[9] J. Lott. Secondary analytic indices. Preprint, 1999.

[10] X. Ma. Functoriality of real analytic torsion form. Preprint, 1999.

[11] X. Ma. Flat duality bundle and the eta form. Preprint, 2000.

[12] X. Ma. Formes de torsion analytique et familles de submersions I. Bull. Soc. Math. France, 128,2000 .

[13] X. Ma. Formes de torsion analytique et familles de submersions II. Asian J. of Math., to appear, 2000. 